\title{
Bayesian estimation of pulsar parameters from gravitational wave data
}

\author{
Réjean J. Dupuis* \\ California Institute of Technology, Pasadena, California 91125, USA \\ University of Glasgow, Glasgow, G12 8QQ, United Kingdom \\ Graham Woan \\ University of Glasgow, Glasgow, G12 8QQ, United Kingdom \\ (Received 24 August 2005; published 16 November 2005)
}

\begin{abstract}
We present a method of searching for, and parametrizing, signals from known radio pulsars in data from interferometric gravitational wave detectors. This method has been applied to data from the LIGO and GEO 600 detectors to set upper limits on the gravitational wave emission from several radio pulsars. Here we discuss the nature of the signal and the performance of the technique on simulated data. We show how to perform a coherent multiple detector analysis and give some insight into the covariance between the signal parameters.
\end{abstract}

DOI: 10.1103/PhysRevD.72.102002

PACS numbers: 04.80.Nn, 02.50.- r

\section{INTRODUCTION}

Several kilometer-scale gravitational wave interferometers are now under construction or actively collecting data with unprecedented sensitivity $[1,2]$. To make the best use of these data, sophisticated analysis methods have been developed to search for astrophysical signals that are doubtless buried in the noise. A promising class of sources of gravitational waves are rapidly rotating, and structurally asymmetric, neutron stars. Several mechanisms have been proposed that could support a varying quadrupolar mass distribution in these neutron stars, and the subsequent continuous emission of gravitational radiation $[3,4]$.

In this paper we present a detailed end-to-end description of an analysis technique which we have developed to infer the parameters of such sources using data from interferometric gravitational wave detectors [5]. Here we test the performance of the technique on simulated data. Whether a signal is clearly present or not, the method can be used to set upper limits on emission strength.

This method was successfully applied first to GEO 600 and LIGO data from their first science run (S1) to set upper limits on the strength of gravitational wave emission from pulsar J1939 + 2134 [6]. The search was modified and expanded to 28 isolated pulsars using data from LIGO's second science run (S2) [7]. This paper investigates the methods used in these two papers and sets performance benchmarks for the algorithm.

Searches for periodic gravitational wave signals from neutron stars are conventionally classed as blind, directed or targeted. A search is blind if no source parameters (such as sky position or spin evolution) are known a priori, so that the size of the parameter space to be explored is maximal. Blind searches represent a computationally demanding problem, and highly efficient analysis techniques must be used [8,9]. Even with these methods, a fully

*Electronic address: rejean@caltech.edu coherent search using many months of data is computationally intractable, and the size of the parameter space has the effect of decreasing the sensitivity of the search, as there is a good chance the noise will imitate a relatively strong source somewhere in the space [10]. Directed (known location) and targeted (known location and phase evolution) searches have smaller numbers of unknown parameters, and vastly smaller parameter spaces, making the detection level lower and increasing their sensitivity to gravitational waves.

Radio pulsars are a particularly interesting class of targeted sources because (i) we can monitor their rotation and make a very good guess at the gravitational waveform they produce and (ii) their locations are known to high precision. In addition, the data pertaining to each pulsar can be restricted to a very narrow spectral window. Real interferometric data are usually contaminated by a large number of instrumental spectral lines, so the effects of these can be reduced significantly by analyzing only those narrow bands containing pulsar data.

This paper is structured as follows: Sec. II describes the nature of the gravitational wave signal that we are expecting from pulsars. Section III describes how we filter and greatly reduce the size of the data set using a semistandard heterodyne technique. In Sec. IV we present the Bayesian methodology used for this analysis and describe the two likelihood functions used in previous work. We demonstrate the performance of the algorithm on simulated data in Sec. V. Section VI concludes the paper with a brief summary.

\section{NATURE OF GRAVITATIONAL WAVE SIGNAL}

Here we summarize the form of gravitational waves emitted from a rotating rigid triaxial pulsar, described in detail in [11]. We take the special case of a triaxial ellipsoid rotating about its principal axis and therefore emitting all its gravitational radiation at twice the rotation frequency. A 
freely precessing neutron star, with its spin and angular momentum axes nonaligned, would also emit at its rotation frequency but is expected to be strongly damped [12]. The regularity of signals from the majority of radio pulsars suggests that most of them are not precessing on a short time scales, if at all.

The gravitational wave amplitude from a triaxial neutron star seen from Earth is

$$
h_{0}=\frac{16 \pi^{2} G}{c^{4}} \frac{I_{z z} f_{\mathrm{r}}^{2}}{r} \epsilon,
$$

where $r$ is the distance to the pulsar, $I_{z z}$ its moment of inertia about the rotation (principal) axis, $f_{\mathrm{r}}$ the rotation frequency of the pulsar, and $\epsilon$ its equatorial ellipticity, defined in terms of its principal moments of inertia as

$$
\epsilon=\frac{I_{x x}-I_{y y}}{I_{z z}} .
$$

The gravitational wave strain on the detector will be frequency modulated, due to the relative motion of the Earth and the pulsar, and amplitude modulated by the antenna pattern of the interferometer. Following [8,11], we can describe this measured signal as

$$
\begin{aligned}
h(t)= & \frac{1}{2} F_{+}(t ; \psi) h_{0}\left(1+\cos ^{2} \iota\right) \cos \Phi(t) \\
& +F_{\times}(t ; \psi) h_{0} \cos \iota \sin \Phi(t),
\end{aligned}
$$

where $F_{+}$and $F_{\times}$are the antenna responses to the + and $\times$ polarizations, respectively, $\psi$ is the polarization angle of the signal (determined by the position angle of the spin axis, projected on the sky), $\iota$ is the inclination of the pulsar with respect to the line-of-sight, and $\Phi(t)$ is the phase of the gravitational wave signal [13].

We choose to time the signal phase evolution $\Phi(T)$ with respect to the solar system barycenter (SSB), which is an inertial reference frame, so that to third-order in barycentric time, $T$,

$$
\begin{aligned}
\Phi(T)= & \phi_{0}+2 \pi\left[f_{\mathrm{s}}\left(T-T_{0}\right)+\frac{1}{2} \dot{f}_{\mathrm{s}}\left(T-T_{0}\right)^{2}\right. \\
& \left.+\frac{1}{6} \ddot{f}_{\mathrm{s}}\left(T-T_{0}\right)^{3}\right],
\end{aligned}
$$

where $\phi_{0}$ is the phase of the signal at a fiducial time $T_{0}, f_{\mathrm{s}}$ is the frequency of the signal $\left(=2 f_{\mathrm{r}}\right), \dot{f}_{\mathrm{s}}$ is the first frequency derivative, and $\ddot{f}_{\mathrm{s}}$ is the second frequency derivative, all at time $T_{0}$. The transformation between the barycentric time $(T)$ and the topocentric time at the detector $(t)$ is

$T=t+\delta t=t+\Delta_{\text {Roemer }}+\Delta_{\text {Shapiro }}+\Delta_{\text {Einstein }}+\Delta_{\text {Binary }}$,

where $\Delta_{\text {Roemer }}$ is the classical Roemer delay, $\Delta_{\text {Shapiro }}$ is the Shapiro delay due to the curvature of space-time near the Sun, $\Delta_{\text {Einstein }}$ is the Einstein delay due to gravitational redshift and time dilation, and $\Delta_{\text {Binary }}$ contains corrections related to the pulsar's orbit, which is zero for isolated neutron stars; see [14] for more details on these terms. However, for pulsars in binary systems this term should include all the classical and relativistic corrections for the shifts in the time of arrival of the signal due to the motion of the source within the binary system. We will not consider binary pulsars in this analysis, but for more details on pulsar timing of binary systems see $[15,16]$, and references therein.

The second term in Eq. (5), the Roemer delay, is the largest component (up to $\sim 8.5 \mathrm{~min}$ ) and due to the motion of the Earth within the solar system. In terms of the Earth's motion it is

$$
\Delta_{\text {Roemer }}=\frac{\mathbf{r}_{d} \cdot \mathbf{k}}{c}+\frac{\left(\mathbf{r}_{d} \cdot \mathbf{k}\right)^{2}-\left|\mathbf{r}_{d}\right|}{2 c d},
$$

where $\mathbf{r}_{d}$ is the position of the detector with regard to the $\mathrm{SSB}, \mathbf{k}$ is a unit vector in the direction of the neutron star, $c$ is the speed of light and $d$ is the distance from the detector to the pulsar. In order to calculate the Roemer delay we need accurate knowledge of the position of the Earth with regard to the SSB. For our barycentering software [17] we use the solar system ephemerides published by the Jet Propulsion Laboratory [18]. The second term in Eq. (6) is the timing parallax. This takes account of the curvature of the wave fronts emitted from the source and is only significant for the closest sources.

The Shapiro delay $\Delta_{\text {Shapiro }}$ is a relativistic correction for the curvature of space-time near the SSB. Since this curvature is not negligible there will be an extra time delay in the arrival of a signal. In principle this delay can be as large as $120 \mu$ s for signals passing near the edge of the Sun and therefore becomes important for the analysis of signals from millisecond pulsars over periods of $\sim 1 \mathrm{yr}$. The maximum contribution from Jupiter however is only $200 \mathrm{~ns}$ and would not affect the sensitivity of a search.

The Einstein delay describes the combined effect of gravitational redshift and time dilation due to the motion of the Earth. This correction takes into account the varying gravitational potential experienced by a clock on the Earth as it follows its elliptical orbit around the Sun. Again this does not significantly affect signal searches.

\section{THE COMPLEX HETERODYNE METHOD}

Current ground-based interferometric detectors have broadband sensitivity to gravitational waves from frequencies of several tens of hertz up to several kilohertz. As a search for a continuous wave signal involves integrating for months or even years, the datasets involved can become very large indeed. However, the signal we are trying to extract in a targeted search is actually contained in only a very narrow frequency band, so accurate knowledge of the spin parameters of the source (from radio or X-ray observations) allows us to reduce the size of this data set considerably. To do this, we perform a complex heterodyne, followed by filtering and resampling of the data, to reduce 
its size by a factor of about $\sim 10^{6}$ without loss of relevant information. Similar techniques have been applied in a wide range of optical, radio and gravitational searches for sinusoidal signals, most notably (in this context) [19].

We choose to perform a complex, slowly evolving, heterodyne on a targeted source to precisely unwind the apparent phase evolution of the source. However, for a signal from a pulsar recorded by an interferometric detector, there is still a time varying component remaining in the heterodyned signal from the antenna response pattern of the interferometer.

Since the source moves through the antenna pattern on a time scale that is much longer than the original periodic signal, after heterodyning we can resample the data with a much reduced sampling rate. In practice the new sampling rate is determined by our wish to monitor variations in the interferometer noise floor, which changes on time scales of minutes, hours, and days. Since we keep both the real and imaginary part of the sample for each minute, our effective bandwidth is $1 / 60 \mathrm{~Hz}$ centered on the heterodyning frequency which is the instantaneous frequency of the signal at the detector.

We take the calibrated output of a gravitational wave detector to be

$$
s(t)=h(t)+n(t),
$$

where $h(t)$ is a gravitational wave signal and $n(t)$ is noise that is stationary over some time period but not necessarily Gaussian. Using Eq. (3) we recast the signal, $h(t)$, to

$$
h(t)=A(t) e^{i \Phi(t)}+A^{*}(t) e^{-i \Phi(t)},
$$

where

$$
A(t)=\frac{1}{4} F_{+}(t ; \psi) h_{0}\left(1+\cos ^{2} \iota\right)-\frac{i}{2} F_{\times}(t ; \psi) h_{0} \cos \iota,
$$

and $A^{*}$ is the complex conjugate of $A$. For a targeted pulsar we assume the frequency and frequency derivative terms are known from electromagnetic observations so that

$$
\phi(t)=\Phi(t+\delta t)-\phi_{0}
$$

can be calculated to high precision. The heterodyning step involves multiplying the data from the interferometer by $e^{-i \phi(t)}$ to give

$$
\begin{aligned}
s_{\text {het }}(t) & =s(t) e^{-i \phi(t)} \\
& =A(t) e^{i \phi_{0}}+A^{*}(t) e^{-i \phi_{0}-2 i \phi(t)}+n(t) e^{-i \phi(t)} .
\end{aligned}
$$

The heterodyning process removes the rotational phase evolution from the term in $A$, although this term will still vary over the day as the source moves through the antenna pattern of the interferometer. The second (upper sideband) term in $A^{*}$ will oscillate at nearly twice the gravitational wave signal frequency.
We then apply a low-pass antialiasing filter to the heterodyned data stream prior to averaging. We note that, because the original time series was real, we lose no independent information by rejecting the upper sideband. We use a series of three third-order infinite impulse response (IIR) Butterworth filters to do this, with frequency cutoffs that can be adjusted to the characteristics of the data. The main requirement is to prevent spectral disturbances from outside our final $1 / 60 \mathrm{~Hz}$ data band being aliased into our calculation of the averaged data. The selection of the IIR filters and the sampling rate ultimately depends on the opposing needs to over-resolve the time scales on which the noise is nonstationary and for a narrow band to avoid nearby spectral lines.

Finally we resample the filtered data to the post-filtering Nyquist rate and average the results (now $s_{\text {het }}^{\prime}$ ) over a minute to form

$$
B_{k}=\frac{1}{M} \sum_{i=1}^{M} s_{\text {het }}^{\prime}\left(t_{i}\right)
$$

where $k$ is the minute index and $M$ is the number of Nyquist samples in 1 min (typically $\sim 100$ ).

In practice there are computational advantages to performing the heterodyning, filtering and resampling process described above in two steps, starting with a fixed heterodyning frequency and a filter that reduce the sampling rate to about $4 \mathrm{~Hz}$. A second (variable) heterodyne can then be performed on the data to remove the Doppler shifts due to the motion of the Earth. The advantage is that the delay corrections between topocentric and barycentric time-ofarrival need only be calculated 4 times, rather than (for LIGO and GEO) 16384 times, per second.

With the high frequency term in Eq. (11) suppressed we have

$$
\begin{aligned}
B_{k}= & \frac{1}{4} F_{+}\left(t_{k} ; \psi\right) h_{0}\left(1+\cos ^{2} \iota\right) e^{i \phi_{0}} \\
& -\frac{i}{2} F_{\times}\left(t_{k} ; \psi\right) h_{0} \cos \iota e^{i \phi_{0}}+n\left(t_{k}\right)^{\prime},
\end{aligned}
$$

where $n\left(t_{k}\right)^{\prime}$ is the heterodyned and averaged complex noise in bin $k$. By the central limit theorem, we would expect the noise, $n\left(t_{k}\right)^{\prime}$, to be well described by a Gaussian distribution, although the width of this distribution may change over time as the detector sensitivity evolves.

The heterodyned gravitational wave signal in this reduced data set depends on the same four unknown parameters in Eqs. (3) and (4): $h_{0}, \psi, \phi_{0}$, and $\iota$, which can be conveniently held as a vector, a. We proceed in the next section by calculating the (Bayesian) probability of the data given these parameters and finally, through the application of Bayes's theorem and marginalization, we obtain posterior probabilities for each of these parameters given the data collected. 


\section{BAYESIAN FORMALISM}

We take a straightforward Bayesian approach for the following analysis and calculate the posterior probability, $p\left(\mathbf{a} \mid\left\{B_{k}\right\}, I\right)$, of the pulsar parameters a given the binned data, $\left\{B_{k}\right\}$. Bayes's theorem tells us that

$$
p\left(\mathbf{a} \mid\left\{B_{k}\right\}, I\right)=\frac{p(\mathbf{a} \mid I) p\left(\left\{B_{k}\right\} \mid \mathbf{a}, I\right)}{p\left(\left\{B_{k}\right\} \mid I\right)},
$$

where $\mathbf{a}$ is the set of parameters inferred from data $\left\{B_{k}\right\}$, given our model $I$, and with likelihood $p\left(\left\{B_{k}\right\} \mid \mathbf{a}, I\right)$. I remains constant throughout the analysis, and will be dropped from the following expressions to avoid clutter. It should of course be noted that all the inferences we make from the data assume this model to be true. Note that the posterior probability distribution given here assumes that a signal is present in the data.

Our prior beliefs in the value of the parameters are held in $p(\mathbf{a})$ and we will use the least informative priors for most of the parameters over their respective ranges. A change in polarization angle of $\pi / 2$ on the sky is equivalent to a change of signal sign (i.e., a change in signal phase, $\phi_{0}$, of $\pi)$, so consistent priors are $\phi_{0}$ uniform over $[0,2 \pi]$ and $\psi$ uniform over $[-\pi / 4, \pi / 4]$. The prior probability density function for cosı is taken as uniform over $[-1,1]$, corresponding to a uniform probability per unit solid angle for the orientation of the spin axis.

The prior probability for $h_{0}$ is more interesting. In principle the prior for $h_{0}$ should reflect all our initial beliefs on the gravitational wave strength, $h_{0}$. If, for example, we truly believe that gravitational wave emission is powered by the loss of kinetic energy from the pulsar (of known spin down rate), and that the moment of inertia of the pulsar is reasonably well constrained, then we should construct a prior that falls away sharply at strain levels which are above those consistent with this spin down upper limit. Of course we know that current detector sensitivities are insufficient to detect such a signal, and as a result the prior would overwhelm the broader likelihood function. We would learn nothing new from the experiment since the posterior probability distribution function (pdf) would largely resemble the prior pdf we chose.

At this stage in gravitational astronomy, a more useful statement would be concerned with what the observations told us that was independent of spin down arguments, and therefore the prior should reflect this greater sense of ignorance. We cannot exclude the prior possibility of $h_{0}=$ 0 , so a fully scale-invariant Jeffreys prior $\left(\propto 1 / h_{0}\right)$ would not be appropriate. However, we are interested in being able to set conservative upper limits on the strength of any signals, and this argues in favor of using a uniform prior for $h_{0}$. A uniform prior favors larger values of $h_{0}$ over smaller values (e.g., the prior probability for the range 0.1 to 1 is 10 times less than for 1 to 10), and represents, for most, an acceptable state of optimistic ignorance. The resulting upper limit for $h_{0}$ will therefore reflect the maximum value that could reasonably be thought of as consistent with the data and has some additional merit because of that. In addition, a posterior based on a uniform prior for $h_{0}$ can be interpreted as a (marginal) likelihood for $h_{0}$ and more easily incorporated into future analyses with other data.

For further discussion on choosing priors in cases, similar to this one, when the level of any signal may be below the sensitivity of the experiment, see [20]. Ultimately, if there is a strong detection the choice of the prior should not play an important role in the results since the likelihood function would be sufficiently strongly peaked to define the posterior. Conversely, if no signal is present at the sensitivity level of the instrument, the prior takes on a greater significance.

The full 4-dimensional posterior pdf contains all the information from our analysis but is difficult to interpret directly. It is therefore useful to reduce the dimensionality by marginalizing (integrating) over the less interesting (nuisance) parameters. The marginal distribution for one parameter can be viewed as a weighted average of all the distributions of this parameter given all the possible combinations of the other parameters. The parameter in which we are most interested is the gravitational wave amplitude $h_{0}$, with a marginal pdf of

$$
p\left(h_{0} \mid\left\{B_{k}\right\}\right) \propto \iiint p\left(\left\{B_{k}\right\} \mid \mathbf{a}\right) p(\mathbf{a}) d \phi_{0} d \psi d \cos \iota,
$$

where the integral is performed numerically, over the full ranges of the nuisance parameters. The pdf for $h_{0}$ can then be normalized trivially.

Even without a detection, placing upper limits on $h_{0}$ can be physically interesting as we are essentially constraining the equatorial ellipticity of the neutron star. We define the upper limit of $h_{0}$ bounding $95 \%$ of the cumulative probability (from $h_{0}=0$ ) as the value $h_{95}$ that satisfies

$$
0.95=\int_{h_{0}=0}^{h_{95}} p\left(h_{0} \mid\left\{B_{k}\right\}\right) d h_{0} .
$$

Note that such a limit can be placed on $h_{0}$ even if most of the probability is to be found some distance from $h_{0}=0$ and a strong signal is detected.

In order to calculate the likelihood function we need to have a model of the signal in the processed data. The model of the signal that we are searching for in the data set, $\left\{B_{k}\right\}$, is obtained by processing the original gravitational wave signal $h(t)$ in the same way that we processed the data to give

$$
\begin{aligned}
y\left(t_{k} ; \mathbf{a}\right)= & \frac{1}{4} F_{+}\left(t_{k} ; \psi\right) h_{0}\left(1+\cos ^{2} \iota\right) e^{i \phi_{0}} \\
& -\frac{i}{2} F_{\times}\left(t_{k} ; \psi\right) h_{0} \cos \iota e^{i \phi_{0}} .
\end{aligned}
$$

We note that the model is complex and that the only time varying component is the antenna pattern of the interferometers. The Nyquist frequency for this signal is well 
below our reduced sampling rate of one $B_{k}$ per minute. In the following two sections we will present two different ways of evaluating the likelihood function depending on whether the variance of the data is known or unknown.

\section{A. Gaussian model-known variance}

Here we give the expression for the likelihood function assuming that we know, or can estimate accurately, the variance of the noise. We assume that the data comprises $n$ samples of a signal, $y\left(t_{k}\right)$ [see Eq. (17)], embedded in complex Gaussian noise $N\left(0, \sigma_{k}\right)$ of zero mean and known overall variance $\sigma_{k}^{2}$, so that

$$
B_{k}=y_{k}+N\left(0, \sigma_{k}\right) .
$$

If the set of $\left\{B_{k}\right\}$ are independent, the likelihood of the data is simply the product of $n$ bivariate normal distributions. Note that the distribution is bivariate because the data are now complex. The real and imaginary parts of the $B_{k}$ 's have independent noise components, each with a variance $\sigma_{k}^{2} / 2$. The likelihood of the parameters is therefore

$$
p\left(\left\{B_{k}\right\} \mid \mathbf{a},\left\{\sigma_{k}\right\}\right)=\left(\sqrt{2 \pi} \sigma_{k}\right)^{-2 n} \exp \left(-\sum_{k=1}^{n} \frac{\left|B_{k}-y_{k}\right|^{2}}{2 \sigma_{k}^{2}}\right) .
$$

This Gaussian model for the likelihood was used for the first GEO 600 and LIGO analysis for signals from pulsar $\mathrm{J} 1939+2134$ [6]. For this analysis, the noise level $\sigma_{k}$ was not known but was estimated for each $B_{k}$ from the noise floor in a $4 \mathrm{~Hz}$ band of data around the signal frequency, assumed stationary for at least $1 \mathrm{~min}$. This gives $240 \times 2$ points contributing to the estimate of the variance, making the uncertainty in the point estimate of $\sigma_{k}$ small enough to be ignored. The procedure is valid as long as the mean noise floor in the band is representative of the noise floor at the signal frequency, if there are no strong contaminating signals in the band and if the noise is sufficiently stationary. Although these requirements were largely met for [6], just one millisecond pulsar was involved in the study and they cannot be expected to be met in general. To address this an alternative model was developed for the $\mathrm{S} 2$ analysis [7].

\section{B. Gaussian model-unknown variance}

In the previous section we evaluated the likelihood function given the noise level, $\sigma_{k}$, for each $B_{k}$. Generally however, the noise level may not be known in advance or may not be well estimated from the data. Here we described the likelihood function appropriate to this situation, which was used in the analysis of the LIGO S2 data [7].

If $\sigma_{k}$ is estimated from a tighter bandwidth, or over a shorter period, fewer data contribute and the uncertainty in its value may be too large to use a point estimate alone. Within our Bayesian framework the standard (and correct) approach is to treat the noise level as another nuisance parameter and marginalize over it, without computing a point estimate at all [21].

We begin by calculating the likelihood of a subset of $m_{j}$ consecutive data points from $\left\{B_{k}\right\}$ which have a constant noise level $\sigma_{j}$. Once we have that expression, we will calculate the global likelihood simply using the product rule assuming that each segment of data is independent. We will again define $n$ to be the total number of data points $B_{k}$ and let $M$ be the number of segments of data that we have assumed have the same noise level, so that

$$
n=\sum_{j=1}^{M} m_{j}
$$

We can write the likelihood of the parameters, based on the $j$ th subset of data and marginalized over $\sigma_{j}$ as

$$
\begin{aligned}
p\left(\left\{B_{k}\right\}_{j} \mid \mathbf{a}\right) & \propto \int_{0}^{\infty} p\left(\left\{B_{k}\right\}_{j}, \sigma_{j} \mid \mathbf{a}\right) d \sigma_{j} \\
& \propto \int_{0}^{\infty} p\left(\sigma_{j} \mid \mathbf{a}\right) p\left(\left\{B_{k}\right\}_{j} \mid \mathbf{a}, \sigma_{j}\right) d \sigma_{j},
\end{aligned}
$$

where $p\left(\sigma_{j} \mid \mathbf{a}\right)$ is the prior for the noise floor and the likelihood $p\left(\sigma_{j} \mid \mathbf{a}\right) p\left(\left\{B_{k}\right\}_{j} \mid \mathbf{a}, \sigma_{j}\right)$ is given by Eq. (19). As $\sigma_{j}$ is a nonzero scale parameter we take a Jeffreys prior, uniform in $\log \sigma_{j}$ :

$$
p\left(\sigma_{j} \mid \mathbf{a}\right) \propto \frac{1}{\sigma_{j}} \quad\left(\sigma_{j}>0\right) .
$$

Our final conclusions would be essentially unchanged if a uniform prior was used instead of this Jeffreys prior [22]. Here we assume that the $\sigma_{j}$ associated with each subset $\left\{B_{k}\right\}_{j}$ is constant over the $m_{j}$ samples. In other words, we assume that the noise level of the interferometer, in a narrow frequency band around the gravitational wave signal, is stationary for this time. However, we allow the noise floor to change between each subset of data $\left\{B_{k}\right\}_{j}$. This allows us to dynamically track the noise floor seen in real interferometric data, which will inevitably vary on some time scale, as the instrumental performance varies. The length of the $j$ th subset, over the which the data is assumed stationary, can also be adjusted to reflect the known time scale of these variations.

Using Eqs. (19), (21), and (22), the likelihood of the parameters based on a subset $\left\{B_{k}\right\}_{j}$ of constant noise $\sigma_{j}$ is

$$
p\left(\left\{B_{k}\right\}_{j} \mid \mathbf{a}\right) \propto \int_{0}^{\infty} \frac{1}{\sigma_{j}^{2 m_{j}+1}} \exp \left(-\sum_{k=k_{1(j)}}^{k_{2(j)}} \frac{\left|B_{k}-y_{k}\right|^{2}}{2 \sigma_{j}^{2}}\right) d \sigma_{j},
$$

where $m_{j}=k_{2(j)}-k_{1(j)}+1$. This reduces to

$$
p\left(\left\{B_{k}\right\}_{j} \mid \mathbf{a}\right) \propto\left(\sum_{k=k_{1(j)}}^{k_{2(j)}}\left|B_{k}-y_{k}\right|^{2}\right)^{-m_{j}},
$$


which is equivalent to a student's $t$-distribution with $2 m_{j}-$ 1 degrees of freedom. Recall that the likelihood derived in Eq. (24) is for a set of $m_{j}$ data points $B_{k}$ with the same $\sigma_{j}$. The joint likelihood of all the $M$ stretches of data, taken as independent, is therefore

$$
p\left(\left\{B_{k}\right\} \mid \mathbf{a}\right) \propto \prod_{j}^{M}\left(\sum_{k=k_{1(j)}}^{k_{2(j)}}\left|B_{k}-y_{k}\right|^{2}\right)^{-m_{j}} .
$$

We note that there is (of course) no explicit reference to the noise level, but the lengths of the stationary intervals, $m_{j}$, can be adjusted to reflect the performance of the detectors.

\section{PERFORMANCE ON SIMULATED DATA}

\section{A. Expected sensitivity}

The analysis described above is optimal (in a Bayesian sense) for the data available from one or more science runs. It is however instructive to examine the long-term performance of the method on a large series of simulated data sets, both to confirm that the average performance complies with our expectations and to ease comparisons with methods based on sampling theory.

To do this, we calculated the $h_{95}$ upper limits from 4000 simulated data sets, of length 10 days, varying the location of the putative source in the sky and the location of the detector in each set. The locations of the sources were picked randomly from a uniform distribution over the sky, and the detector locations were the GEO 600 and the two LIGO sites.

From these we can express the average 95\% upper limit $\left\langle h_{95}\right\rangle$ as a function of observation time $T$ and single-sided

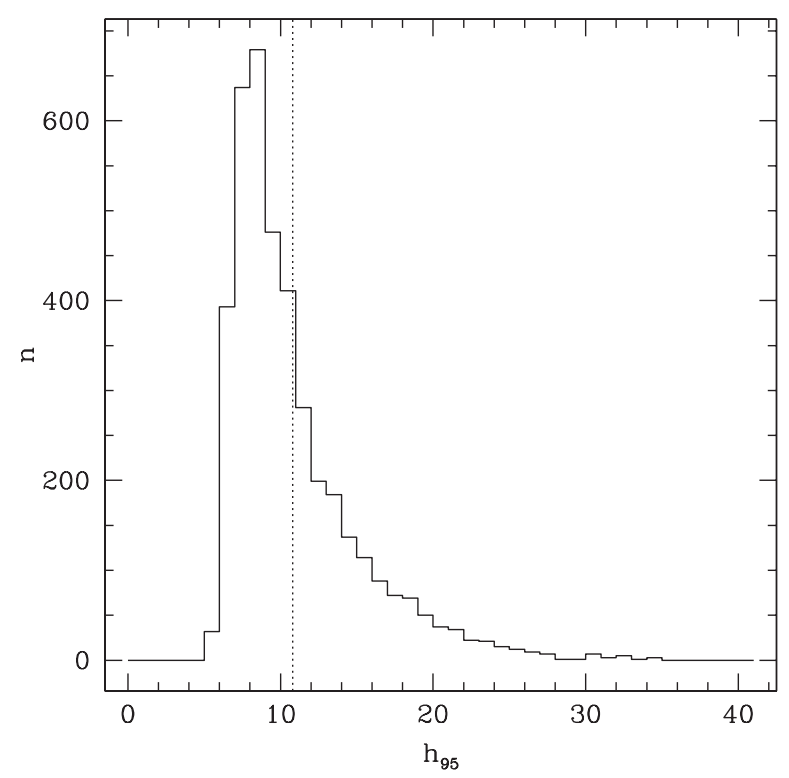

FIG. 1. Distribution of $95 \%$ upper limits on $h_{0}$ for 4000 simulations, using sources randomly located on the sky with $S_{n}(f) / T=1$. noise power spectral density, $S_{n}(f)$. Empirically, from these simulations,

$$
\left\langle h_{95}\right\rangle=(10.8 \pm 0.2) \sqrt{S_{n}(f) / T},
$$

where the range accounts for the location of the detector. Figure 1 shows the distribution of $h_{95}$ that contributed to this, for $S_{n}(f) / T=1$. Note that the width and skew of the distribution are relatively large, so the actual upper limit from an observing run could reasonably be up to a factor of 2 larger than $\left\langle h_{95}\right\rangle$.

\section{B. Combining data from a network of detectors}

Several gravitational wave detectors are currently collecting data, and ideally we should be able to use the observations from all detectors in a coherent manner in order to draw the best possible inference about the source parameters. In a Bayesian analysis all observations enter via the likelihood function. Assuming that the noise from each interferometer is independent, by the product rule the global likelihood is simply the product of the individual likelihoods. For example, by combining observations from GEO 600 and the three LIGO interferometers, we would get

$$
p\left(\left\{B_{k}\right\}_{\text {Joint }} \mid \mathbf{a}\right)=\prod_{i=1}^{4} p\left(\left\{B_{k}\right\}_{i} \mid \mathbf{a}, i\right)
$$

where the product is over the $4 \mathrm{~km}$ Hanford interferometer (H1), the $2 \mathrm{~km}$ Hanford interferometer $(\mathrm{H} 2)$, the $4 \mathrm{~km}$ Livingston interferometer (L1), and GEO 600.

This likelihood contains all the information on the source parameters that is contained in the data, optimally combining the data from all the interferometers in a coherent way. Note that the observation periods can be different and so can the sensitivity of the detectors, although for detectors with very different sensitivities, this will closely approximate the likelihood based on just the most sensitive instrument.

For illustration, we generated four sets of data of 10 days length with Gaussian noise ( $\mu=0$ and $\sigma=1$ ) as if from GEO 600 and the three LIGO interferometers. As these IFOs are modeled as having the same sensitivity, we would expect the coherent results to be approximately $\sqrt{4}$ times tighter than the individual results (distinguished from a factor of 4 increase in observing time only by the differing antenna patterns of the instruments). The four posterior pdfs for each detector, as well as the joint multidetector posterior pdf for $h_{0}$, are shown in Fig. 2. The individual 95\% upper limits are 0.15 for GEO 600, 0.16 for $\mathrm{H} 1,0.18$ for $\mathrm{H} 2$, and 0.13 for $\mathrm{L} 1$ giving an average of 0.155 . The combined $95 \%$ upper limit, on the other hand, is 0.08, which is indeed approximately a factor of 2 better than the average of the limits from the individual detectors. This technique was first applied to real gravitational wave data in [7]. The equivalent multidetector analysis using the 


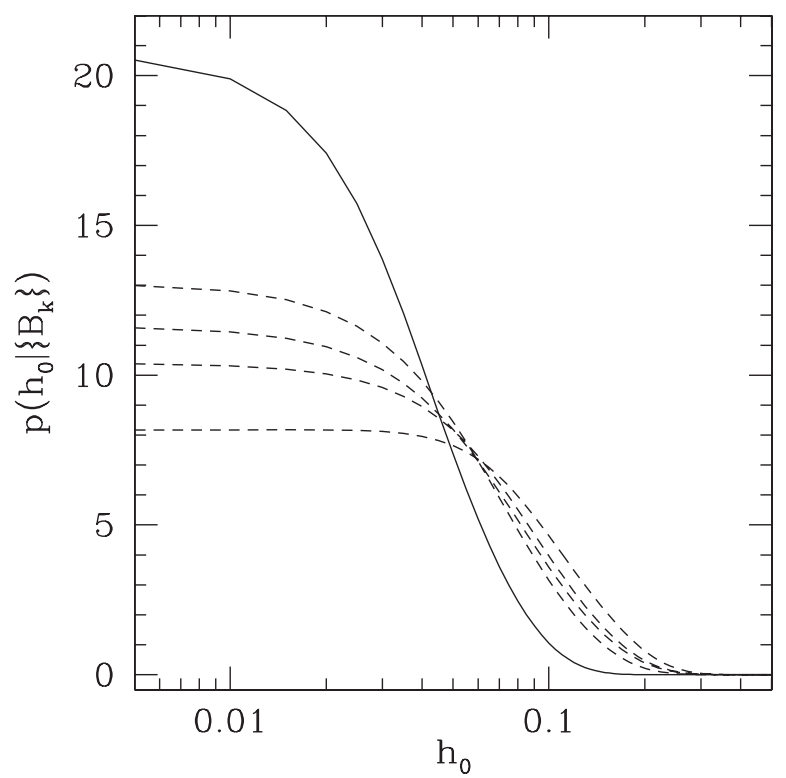

FIG. 2. Multidetector posterior pdfs with simulated data. The solid line represents the joint marginalized posterior pdf for $h_{0}$ using the data from four separate interferometers. The dashed lines are the corresponding pdfs from the individual detectors.

$\mathcal{F}$-statistic method [8] has recently been developed by Cutler and Schutz [23].

It is important to realize that the posterior curve derived from a particular observation represents a probabilistic statement about the value of $h_{0}$ based on the data in hand and may, if we are unlucky, be wildly at odds with the truth. As a result the upper limit derived from one instrument alone will occasionally be lower than that from the coherent combination of instruments.

\section{Effects of changing the noise level}

The widths of the marginal posteriors depend on both the level of the noise and the covariance of the parameters. Here we demonstrate the noise dependence by analysing three sets of data with different, sometimes modulated, noise variances. Each data set corresponds to 10 days of observations. The first contains Gaussian noise with $\mu=0$ and $\sigma=1$. For the second data set, the noise level alternates each $30 \mathrm{~min}$ between $\sigma=10$ and $\sigma=1 /(2 \sqrt{2})$. For the third data set, the noise level alternates each $30 \mathrm{~min}$ between $\sigma=100$ and $\sigma=1 /(5 \sqrt{2})$. Two time series plots showing representative stretches of data from the first and second sets are shown Fig. 3.

For this test, we repeated and averaged the posterior pdfs for 100 generations of the data sets described above. The average marginalized posterior pdfs for $h_{0}$ are shown in Fig. 4.

Using the $66 \%$ upper limit on $h_{0}$ to characterize the width of the pdfs we have $h_{0}<0.095$ for case $1, h_{0}<$ 0.047 for case 2 , and $h_{0}<0.019$ for case 3 . The second posterior is narrower than the first by a factor of 2.02 and
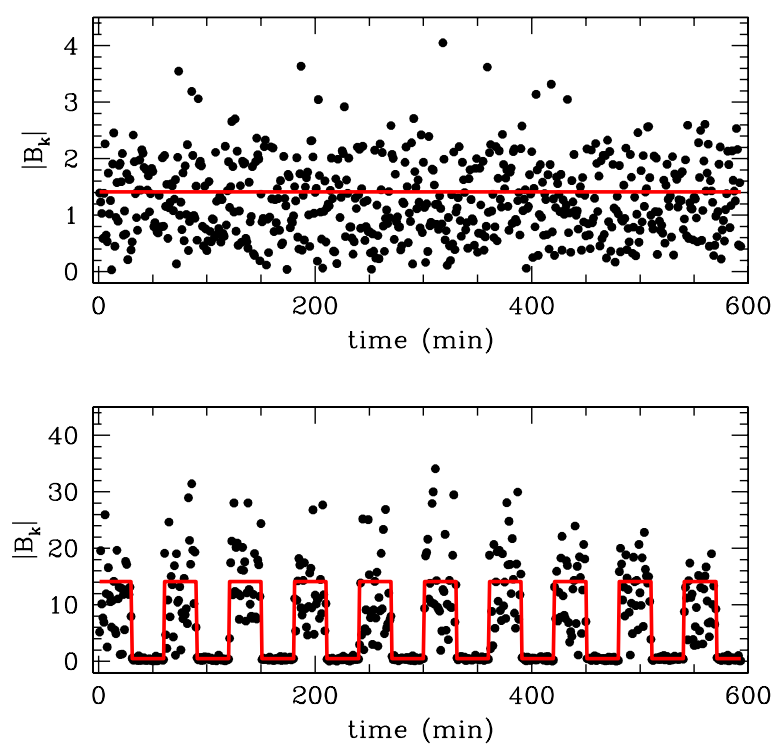

FIG. 3 (color online). Time series of $B_{k}$ showing the effect of a changing noise level. The dots are the $\left|B_{k}\right|$ and the line represents the variance of the $B_{k}$ 's. The top figure represents the first data set with constant variance and the bottom figure represents the second data set with alternating variance.

the third by a factor of 5.05. For no signal, these results are roughly what we would expect: in the two cases with alternating noise levels, about half of the data has very low sensitivity compared to the other half, and we can assume this noisy half does not play a significant part in the posterior. Therefore these cases are approximately equivalent to a continuous observation at the greater sensi-

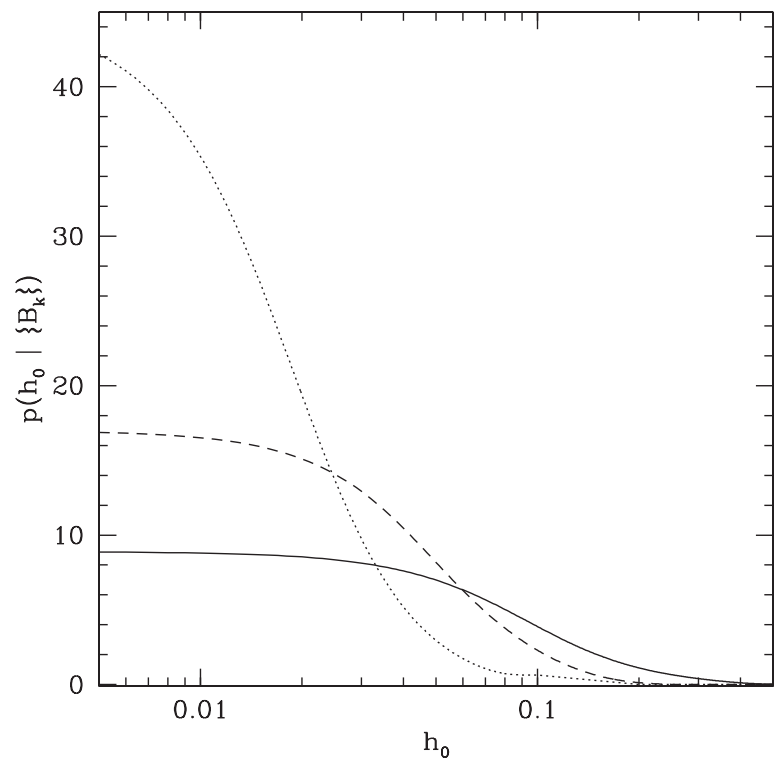

FIG. 4. Averaged marginalized posterior pdfs for $h_{0}$ for three scenarios: constant unit variance (solid line), alternating noise level between $\sigma=10$ and $\sigma=1 /(2 \sqrt{2})$ (dashed line), and alternating noise level between $\sigma=100$ and $\sigma=1 /(5 \sqrt{2})$ (dotted line). 
tivity level but for half the full observing period, and the reduced time lowers the sensitivity by $\sqrt{2}$. Thus compared to the first case with constant noise, we would expect the two sets with alternating noise levels to have widths which are narrower by factors of about 2 and 5 , which is what we see.

\section{Covariance between parameters}

To illustrate the covariance between the signal parameters we generated a 10 day time series containing a signal with the following parameters and Gaussian noise of unit variance: $h_{0}=0.25, \cos \iota=0.1, \phi_{0}=180^{\circ}, \psi=0.0^{\circ}$. It is clear from the emission model that if $\cos \iota \neq 0, h_{0}$ and $\cos \iota$ are strongly anticorrelated, as are $\psi$ and $\phi_{0}$. The correlation can be seen clearly in the probability density contour plots in Fig. 5.

The covariance between $\cos \iota$ and $h_{0}$ contributes strongly to the overall width of the marginal pdf of $h_{0}$, making the precise value of $h_{0}$ somewhat difficult to de-
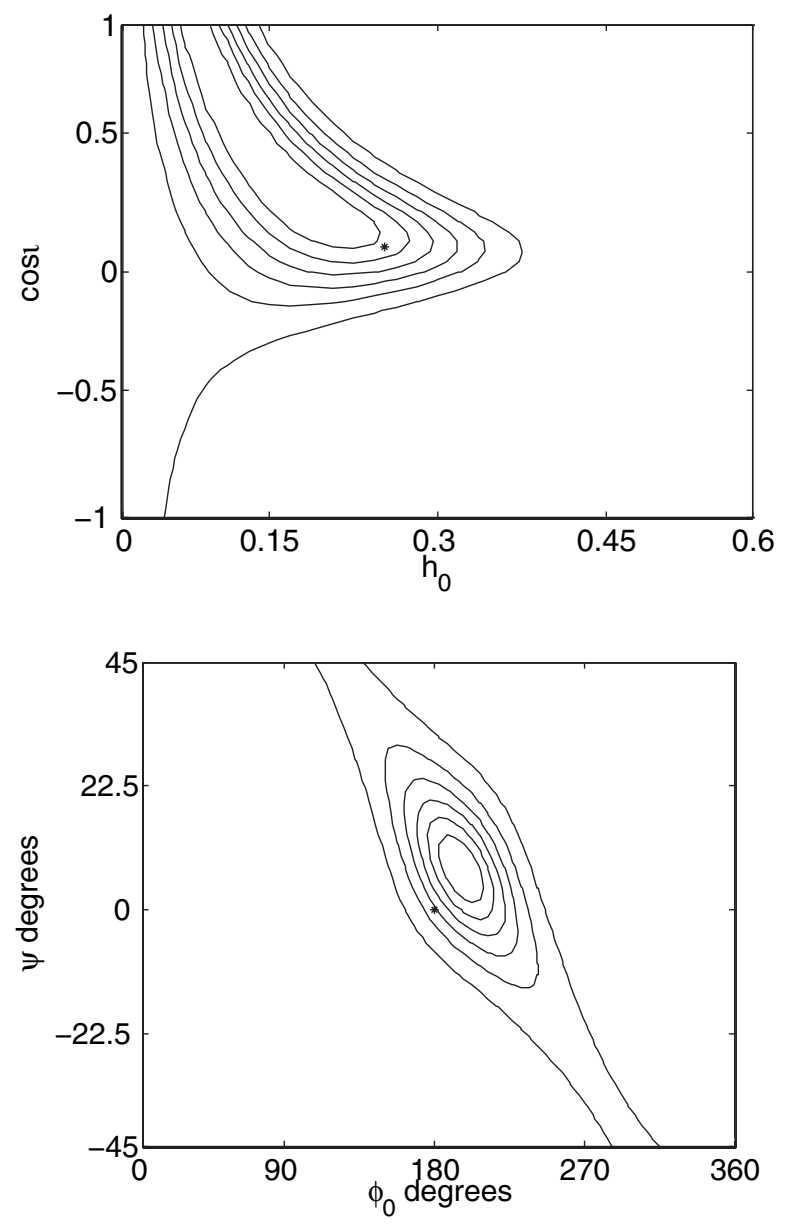

FIG. 5. Equally spaced contours of constant probability density for (top) the joint posterior probability of $\cos \iota$ and $h_{0}$ and (bottom) $\psi$ and $\phi_{0}$. The marker indicates the location of the simulated signal.

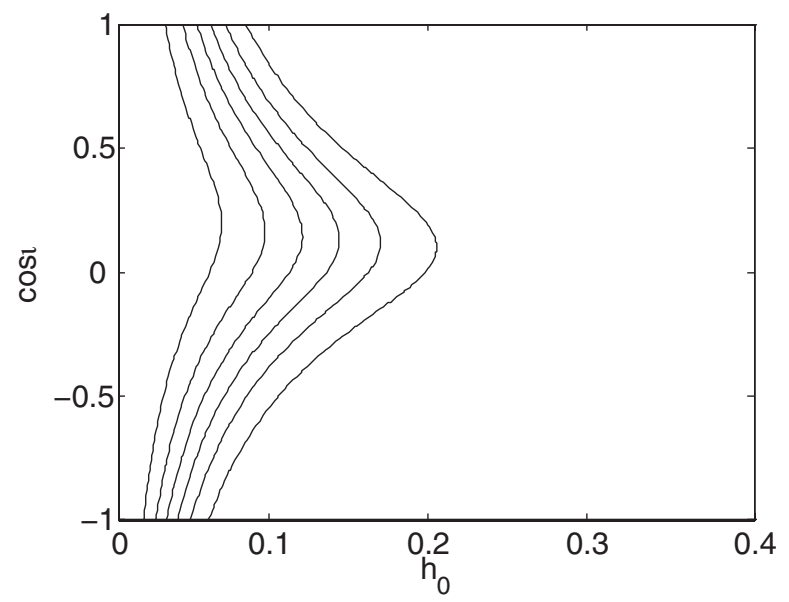

FIG. 6. Equally spaced contours of constant probability, showing the covariance between $h_{0}$ and $\cos \iota$, for Gaussian noise with no signal but a uniform prior for $h_{0}$.

termine even under conditions of relatively high signal-tonoise ratio. The correlation between $h_{0}$ and $\cos \iota$ is also visible when no gravitational wave signal is injected in the data $\left(h_{0}=0\right)$, largely because of our choice of a uniform prior for $h_{0}$ which holds out high hopes for a strong signal in the data (Fig. 6). When no such signal is seen, this is interpreted as an indication that the pulsar is oriented unfavorably and the posterior probability slightly increases around $\cos \iota=1$, where only the " + " polarization is present.

\section{CONCLUSIONS}

In this paper we have presented an end-to-end Bayesian method of searching for, and parametrizing, gravitational waves from known pulsars. The method involves processing the raw data to reduce the number of samples required for the analysis. We calculated the likelihood function for given model parameters from the decimated data, so reducing computational requirements. The algorithm has been validated by retrieving the correct signal parameters from simulated data. We have also shown than it is easily adapted to deal with a network of detectors.

This methodology was initially developed for targeted searches with known locations and spin evolutions of the sources. Further work has now been done studying the feasibility of expanding the numbers of parameters by taking advantage of Monte Carlo Markov chain techniques [24]. These techniques are required when the number of unknown parameters is significantly increased, when the method presented here would be too computationally intensive. In a future paper we will address how this method has been adapted to search for gravitational emission from pulsars in binary systems. The algorithm presented in this paper, with the binary modification, is currently being applied to GEO 600 and LIGO data from the S3 and S4 science runs. 


\section{ACKNOWLEDGMENTS}

The authors would like to thank the LSC Pulsar Group for useful discussions. This work was supported by the Natural Sciences and Engineering Research Council of Canada, Universities U.K., and the University of
Glasgow. R. J.D. would also like to acknowledge funding from the National Science Foundation. This document has been assigned LIGO Laboratory document No. LIGOP050046-00-Z.
[1] B. Abbott et al. (LIGO Scientific Collaboration), Nucl. Instrum. Methods Phys. Res., Sect. A 517, 154 (2004).

[2] B. Caron et al. (VIRGO Collaboration), Nucl. Phys. B, Proc. Suppl. 54, 167 (1997).

[3] C. Cutler, Phys. Rev. D 66, 084025 (2002).

[4] B. Owen, astro-ph/0503399 [Phys. Rev. Lett. (to be published)].

[5] R. J. Dupuis, Ph.D. thesis, University of Glasgow, 2004.

[6] B. Abbott et al. (LIGO Scientific Collaboration), Phys. Rev. D 69, 082004 (2004).

[7] B. Abbott et al. (LIGO Scientific Collaboration), Phys. Rev. Lett. 94, 181103 (2005).

[8] P. Jaranowski, A. Królak, and B. F. Schutz, Phys. Rev. D 58, 063001 (1998).

[9] B. Krishnan, A. M. Sintes, M. A. Papa, B. F. Schutz, S. Frasca, and C. Palomba, Phys. Rev. D 70, 082001 (2004).

[10] P. R. Brady, T. Creighton, C. Cutler, and B. F. Schutz, Phys. Rev. D 57, 2101 (1998).

[11] M. Zimmermann and E. Szedenits, Phys. Rev. D 20, 351 (1979).

[12] D. I. Jones and N. Andersson, Mon. Not. R. Astron. Soc. 331, 203 (2002).

[13] Authors do not agree on whether one should work in terms of signal phase or rotational phase in these expressions. We use the convention of [6,7], so that $\Phi=\Phi_{\text {sig }}=2 \Phi_{\text {rot }}$.

[14] D. C. Backer and R.W. Hellings, Annu. Rev. Astron. Astrophys. 24, 537 (1986).

[15] J.H. Taylor and J. M. Weisberg, Astrophys. J. 345, 434 (1989).

[16] J. M. Weisberg and J.H. Taylor, astro-ph/0407149, 2004.

[17] The timing routines that we used are available in the LIGO Analysis Library available at http://www.lsc-group.phys. uwm.edu/daswg/projects/lal.html

[18] http://ssd.jpl.nasa.gov/horizons.html

[19] T. M. Niebauer, A. Rudiger, R. Schilling, L. Schnupp, W. Winkler, and K. Danzmann, Phys. Rev. D 47, 3106 (1993).

[20] G. D'Agostini, physics/9906048, 1999.

[21] G. L. Bretthorst, Bayesian Spectrum Analysis and Parameter Estimation, Lecture Notes in Statistics (Springer-Verlag, Berlin, 1988), Vol. 48.

[22] D. Sivia, Data Analysis: A Bayesian Tutorial (Oxford University Press, 1996).

[23] C. Cutler and B.F. Schutz, Phys. Rev. D 72, 063006 (2005).

[24] N. Christensen, R. J. Dupuis, G. Woan, and R. Meyer, Phys. Rev. D 70, 022001 (2004). 